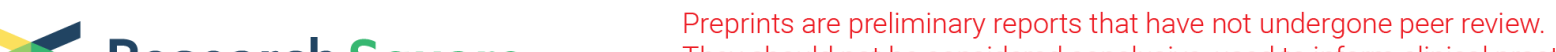 Research Square They should not be considered conclusive, used to inform clinical practice, or referenced by the media as validated information.
}

\section{Seq-SymRF: A random forest model predict potential miRNA-disease associations based on information of sequences and clinical symptoms}

Jinlong Li

Guangdong Pharmaceutical University

Xingyu Chen

Guangdong Pharmaceutical University

Qixing Huang

Guangdong Pharmaceutical University

Yang Wang

Sun Yat-Sen University

Yun Xie

Guangdong Pharmaceutical University

Zong Dai

Sun Yat-Sen University

Xiaoyong Zou ( $\nabla$ ceszxy@mail.sysu.edu.cn )

Sun Yat-Sen University https://orcid.org/0000-0002-9481-9687

Zhanchao Li

Guangdong Pharmaceutical University

\section{Methodology article}

Keywords: Clinical symptom, Disease, MiRNA, MiRNA-disease associations, Random forest

Posted Date: December 18th, 2019

DOI: https://doi.org/10.21203/rs.2.19166/v1

License: (c) (1) This work is licensed under a Creative Commons Attribution 4.0 International License. Read Full License

Version of Record: A version of this preprint was published at Scientific Reports on October 21st, 2020. See the published version at https://doi.org/10.1038/s41598-020-75005-9. 


\section{Abstract}

Background: MicroRNA (MiRNA) plays a vital role in biological processes and closely relate with various human diseases. Research on the miRNA-disease associations contributes to the prevention, diagnosis and treatment of diseases, as well as the identification of new drugs and the discovery of lead compounds. Since traditional experiment methods are time-consuming and expensive, it is necessary to develop an efficient and accurate theoretical approach to identify potential miRNA-disease associations.

Results: In this study, a sequence- and symptom-based random forest classifier model (Seq-SymRF) was constructed to identify the potential associations between miRNA and disease. Compared with existing methods, features derived from only sequence information were used to characterize miRNA. More importantly, clinical symptoms were utilized to represent disease. Moreover, the clustering method by calculating the Euclidean distance was implemented to construct the reliable negative sample. Based on the five-fold cross-validation, the model achieved the accuracy, specificity, sensitivity, precision and Matthews correlation coefficient of $98.00 \%, 99.43 \%, 96.58 \%, 99.40 \%$ and 0.9604 , respectively. The areas under receiver operating characteristic curve and precision recall curve were 0.9967 and 0.9975 , respectively. Additionally, case study was implemented with leukemia, breast neoplasms and hsa-mir-21. Most of top-25 predicted disease-related miRNAs (36/50 for leukemia; 33/50 for breast neoplasms) and 32 of top-50 predicted miRNA-related diseases were verified by literature and dbDEMC database.

Conclusion: We proposed a new method, named Seq-SymRF, for predicting miRNA-disease associations, which could be regarded as a powerful high-throughput virtual screening tool for drug research and development.

\section{Background}

MiRNA is a small non-coding RNA with a length of about 22 bases, which affects biological processes by regulating gene expression. Generally, it binds to the 3 ' untranslated region (UTR) of the target mRNA through sequence-specific base pairing, thereby inhibiting the expression of the target mRNA [1]. Additionally, some studies have shown that miRNA also act as positive regulators [2]. In the recent years, various biological experiments have verified that miRNA plays an important role in many significant biologic processes, including cell proliferation [3], development [4], differentiation [5], apotheosis [6], metabolism [7], aging [8], signal transduction [9], viral infection [10] and so on. For example, high expression of miR-21 has been linked to the development of various cancers [11]. In view of this discovery, Yang et al., [12] extracted Skullcapflavone I from Scutellaria baicalensis Georgi (Wogon) to down-regulate miR-21 expression and treated lung cancer. Therefore, the development of miRNA inhibitors may be a research direction for the design of anticancer drugs.

Since the first two miRNAs lin-4 [13] and let-7 [14] were discovered, about 35,000 miRNAs have been found in different species. Information about these miRNAs was collected in miBase database [15]. Due to the huge number of miRNAs and diseases, the use of traditional experimental method is undoubtedly 
quite time-consuming and expensive for identifying the associations between miRNAs and diseases. Fortunately, with the establishment of more and more biological databases, it is more convenient and effective to develop computational methods for inferring associations between miRNAs and diseases.

In the past decades, a large number of computational models have been developed to predict the potential associations between miRNAs and diseases. These models are mainly based on the assumption that functionally related miRNAs tend to participate in phenotype-like diseases, and vice versa. Those existing approaches can be divided into four categories [16]: (1) score function-based (2) complex network-based, (3) machine learning-based and (4) multiple biological information-based.

For the score function-based methods, Jiang et al., [17] introduced a network computational approach by integrating the functional network, human phenome-miRNAome network and miRNA-disease associations network. However, the method strongly relies on predicted miRNA-target interactions which have high rates of false-positive and false-negative. Chen et al., [18] constructed a model of within and between score for miRNA-disease associations identification (WBSMDA) based on the functional similarity, semantic similarity and Gaussian interaction profile kernel similarity of miRNAs and diseases. In the model, each miRNA-disease association pair obtains a score, the miRNA is more likely related with the disease if corresponding score is higher.

For the complex network-based techniques, Chen et al., [19] presented a random walk-based computational model, random walk with restart for miRNA-disease association (RWRMDA), for inferring novel human miRNA-disease associations. Unfortunately, it is unable to identify new diseases without known associated miRNAs. Subsequently, Xuan et al., [20] proposed the model of miRNAs associated with diseases prediction (MIDP) by implementing random walk on the miRNA-disease bilayer network. Further, Chen et al., [21] infer the potential association by creating a model of heterogeneous graph inference for miRNA-disease association prediction (HGIMDA) based on the combination of known miRNA-disease associations network, miRNA similarity network and disease similarity network. You et al., [22] have developed a novel and effective path-based computational model for identifying miRNAdisease associations (PBMDA) through integrating known human miRNA-disease associations, miRNA similarity and disease similarity. They summed up the path score between a miRNA and disease to calculate the association score for identifying potential associations.

For the machine learning-based methods, Xu et al., [23] constructed a miRNA target-dysregulated network (MTDN). Then, they represented miRNAs by using four-dimension features and employed support vector machine to predict the associations. However, their method only achieved a satisfied result in prostate cancer. Chen et al., [24] developed a semi-supervised computational model to recognize miRNA-disease associations (RLSMDA). They constructed two optimal classifiers 'FM' and 'FD' for miRNA and disease respectively, and combined the two classifiers to infer the potential associations.

For the multiple biological information-based models, Mørk et al., [25] took the protein information into account to infer miRNA-protein-disease associations (miRPD). Similarly, Shi et al., [26] introduced a 
prediction model that mainly considered the associations between miRNA targets and disease genes on the protein-protein interaction network to inferring miRNA-disease associations.

Most of the methods mentioned above characterize each miRNA by calculating its functional similarity with other miRNAs. Even some methods claim that they can be applied to new miRNAs. However, there are still some improvements in functional similarity. For a new discovery miRNA, we first need to know its expression value for calculating similarity with other miRNAs. Besides, a lot of models also need to calculate the Gaussian interaction profile kernel similarity based on the association with different diseases. This characterization of miRNAs leads to a heavy dependence on other miRNAs or diseases. Consequently, most of the prediction methods cannot be effectively applied to newly discovered miRNAs. In fact, sequence is the most intuitive and objective information for each miRNA, because every miRNA has its own distinct sequence. Thus, we extract the features from miRNA sequence to characterize miRNA.

As with miRNA, most methods used semantic similarity proposed by Wang et al. [27] to characterize disease. Based on the MeSH database classification system, each disease is described as a directed acyclic graph and has a semantic value. Although the $\mathrm{MeSH}$ is a rigorous database, it is still somewhat subjective in terms of disease classification. Therefore, it is imperfect and inadequate to calculate the disease semantic similarity based on the MeSH. For disease, clinical symptom is one of the most intuitive features. Community health professionals and general practitioners derive most of their knowledge of the symptoms of individual diseases from hospital-based observations [28]. Compared with traditional method, using symptom as disease feature can contain more information of medicine and is able to characterize disease more reasonably.

Meanwhile, in virtue of no database to collect negative samples, almost all existing methods randomly combine miRNAs and diseases and treat them as negative samples if they are excluded from the database. Because of this reason, the random choice of negative samples will cause a high false negative rate and affect the predictive performance of the model. Therefore, it is necessary to apply a new strategy to select negative samples. In view of these reasons, a novel computational method is proposed to identify potential miRNA-disease associations. In the approach, miRNA and disease are characterized by using features derived from the sequence information and clinical symptoms, respectively. Negative examples are obtained by using clustering method based on calculating the Euclidean distance. Random forest algorithm is utilized to construct identification model. The prediction performance of the current method is evaluated by using the five-fold cross-validation and the practical application performance is also assessment by utilizing some case studies.

\section{Results}

\section{Redundancy of the benchmark dataset}


In order to examine the redundancy of the benchmark dataset, we calculated the similarity of any two miRNAs, diseases and miRNA-disease association pairs, respectively. For two miRNAs, we performed sequence alignment by using CD-HIT software [29] to calculate similarity. For two diseases, we calculated the Jaccard coefficient to evaluate similarity based on the disease symptoms feature vector. For two miRNA-disease association pairs $\mathrm{m}(\mathrm{l})-\mathrm{d}(p)$ and $\mathrm{m}(\mathrm{j}) \mathrm{d}(q)$, their similarity (mDPS) can be calculated according to the Eq. 1:

[Due to technical limitations, the formula could not be displayed here. Please see the supplementary files section to access the formula.]

Where, $S m(i, j)$ and $S d(p, q)$ means the similarity of two miRNAs $\mathrm{m}(i)$ and $\mathrm{m}(j)$ as well as two diseases $\mathrm{d}(p)$ and $\mathrm{d}(q)$.

Similarity values of any two miRNAs, diseases and miRNA-disease association pairs were shown in Fig. 1(A), (B) and (C), respectively. Meanwhile, statistical results of similarity values were shown in Fig. 1(D). For miRNAs, their similarity mainly concentrated in [0.4, 0.5) (value higher than or equal to 0.4 and lower than 0.5 , the same below), accounting for $70.88 \%$ and the proportion of interval $[0.2,0.3),[0.3,0.4),[0.5,0.6),[0.6,0.7),[0.7,0.8)$, $[0.8,0.9)$ and $[0.9,1.0)$ is $0.14 \%, 0.89 \%, 19.56 \%, 0.22 \%, 15.45 \%, 2.11 \%, 0.32 \%$ respectively. Similarity values of any two miRNAs are not included in the range of $[0,0.1)$ and $[0.1,0.2)$. The similarity value between any two diseases is usually low. The $81.98 \%$ and $15.45 \%$ of all similarity values are located in the range of $[0,0.1)$ and $[0.1,0.2)$. Only $2.11 \%, 0.32 \%, 0.0698 \%, 0.0471 \%, 0.0070 \%$ and $0.0087 \%$ concentrated in the range of $[0.2$, $0.3),[0.3,0.4),[0.4,0.5),[0.5,0.6),[0.6,0.7)$ and $[0.7,0.8)$. And, similarity value of any two diseases is always lower than 0.8 .

The distribution of miRNA-disease associations similarity values is more dispersed. The $62.75 \%$ and $32.76 \%$ of associations similarity values concentrated in the range of $[0.2,0.3)$ and $[0.3,0.4)$. The $0.27 \%, 2.15 \%, 0.55 \%, 0.0969 \%, 1.41 \%, 0.0135 \%$ and $0.0017 \%$ are located in the range of $[0.1,0.2),[0.4,0.5),[0.5,0.6),[0.6,0.7),[0.7,0.8),[0.8,0.9)$ and 
$[0.9,1.0)$, respectively. These results indicate that the constructed benchmark dataset has a low redundancy, miRNA sequences are diverse and diseases fall into different categories.

[Figure 1]

\section{Performance evaluation}

To evaluate the identification performance of model, five-fold cross-validation was utilized based on the benchmark dataset, which was divided into 5 parts, each part would be treated as test set in turn and other 4 parts would be treated as training set. We repeated 10 times for each evaluation to minimize the performance difference.

We adopt several evaluation metrics: accuracy (Acc), specificity (Spe), sensitivity (Sen), precision (Pre) and Matthews correlation coefficient (Mcc), the areas under receiver operating characteristic curve (AUROC) and precision recall curve (AUPRC) to assess prediction performance.

\section{Optimization of model parameter}

Random forest is a classifier with multiple decision trees, and the numbers of decision trees and predictor variables at each decision split have an important impact on prediction performance. Therefore, prediction performance of constructed model was optimized based on the grid search strategy, in which change the tree number from 100 to 1000 with interval 100 and vary number of features selected at each split from $2^{1}, 2^{2}, \ldots$, to $2^{5}$ and a default value (i.e. square root of the total number of variables). The Acc, Sen, Spe, Pre, Mcc, as well as AUROC and AUPRC for each combination of tree number and selected variable number were listed in supplement Table $1 \sim 7$.

From results, we can see that the number of trees has less impact on model performance. When the number of trees is determined, the model can obtain better performance as the number of selected feature increases. And, the performance remains stable when the number of selected variables is greater than the default value. Moreover, it takes more time to train the model when the numbers of trees and selected variables are larger. Therefore, 
the optimal model was constructed by setting the number of trees and choose feature as 100 and 22, respectively. Finally, the optimized model achieved the average Acc of 92.27\%, Spe of $92.93 \%$, Sen of $91.62 \%$, Pre of $92.85 \%$, and Mcc of 0.8456 , respectively. The corresponding AUROC and AUPRC were 0.9756 and 0.9787, respectively.

\section{Performance comparing of different negative examples selection strategy}

In order to demonstrate the reliability of the current negative sample selection strategy, we compared it with the random selection strategy. For better comparison, the result of two selection strategy was shown in Fig. 2. When the negative sample was randomly selected, the valudes of Acc, Spe, Sen, Pre, Mcc, AUROC and AUPRC were 83.67\%, 82.93\%, $84.40 \%, 83.17 \%, 0.6733,0.9184$ and 0.9072 , respectively. It is $8.60 \%, 10.00 \%, 7.22 \%$, 9.68\%, 17.23\%, 5.72\%, 7.15\% lower than current method, respectively. Further, we calculated the relative standard deviation (RSD). For the current method, the RSD of Acc, Spe, Sen, Pre and Mcc is $0.28 \%, 0.31 \%, 0.24 \%, 0.32 \%$ and $0.60 \%$, respectively. These values are always lower than those of the random selection strategy $(0.43 \%, 0.53 \%, 0.41 \%$, $0.48 \%$ and $1.08 \%)$. The results demonstrate that the selection of negative sample in the current method is effective and reliable. The lower RSD also prove that current selection strategy can make model more stable.

[Figure 2]

\section{The reliability of negative sample}

In this part, we further construct five reliable negative sample datasets based on the different threshold (1.5AOD, 1.0AOD, 0.8AOD, 0.6AOD and 0.5AOD) to investigate the impact on the performance of the model (nAOD represents $n \times A O D)$. Results of different threshold were shown in Fig. 3. We can see that the performance of the model decrease gradually with the decrease of the threshold. When the threshold is lower than 0.5AOD, there is no performance difference between the current negative samples selection strategy and the random negative samples selection method. We can conclude that the reliability of 
negative samples has a positive impact on the prediction performance. Finally, we chose 1.5AOD as threshold to select reliable negative sample

[Figure 3]

\section{The proportion of positive and negative samples}

In random forest models, the ratio between positive samples and negative samples also affects the model performance. To further explore this effect, we chose five different ratios to construct five training datasets. By increasing the number of negative samples, we get five training datasets with the ratio in $1: 1,1: 2,1: 3,1: 5$ and 1:10 respectively. Results of five-fold cross-validation test were shown in Fig. 4 (In this section, the selecting threshold of reliable negative samples was set to 1.0AOD to ensure that there are sufficient negative samples). As the number of negative samples increases, Acc, Spe and Pre rise slowly, Mcc and AUROC fluctuate randomly, AUPRC decreases continuously. It is worth noting that Sen decreases significantly when the ratio increases from 1:1 to 1:10. Because the purpose of our current research is to identify potential positive samples, the optimal ratio choice of positive and negative samples was set to $1: 1$

\section{[Figure 4]}

\section{Prediction capability for new miRNA}

Identifying potential miRNAs related to known diseases has an important role in drug target discovery and new drug development. To further verify the prediction capability of our method for disease-related potential miRNAs, we remove the miRNA from our dataset if the similarity is higher than a specific threshold. Here, four non-redundant datasets were constructed based on the different thresholds: 0.9, 0.8, 0.7 and 0.6 (When the threshold is lower than 0.6, the number of miRNAs is so small that lost its statistical significance). Results of five-fold cross-validation test based on the various non-redundant datasets were listed in Table 1. For the four non-redundant datasets, AUROC and AUPRC maintain stability at about 0.9962 and 0.9970 . When the threshold is higher than 0.6, Acc, Sen, Spe, Pre and Mcc have a smaller fluctuations range (<1\%). In summary, these results reveal 
that our method has a better robustness and insensitive for the redundancy of dataset, and has outstanding performance for identifying disease-associated potential miRNA.

Table 1 The results of five-fold cross-validation test from the different non-redundant datasets

\begin{tabular}{llllllll}
\hline Threshold & Acc(\%) & Sen(\%) & Spe(\%) & Pre(\%) & Mcc & AUROC & AUPRC \\
\hline 0.9 & 97.98 & 96.34 & 99.61 & 99.60 & 0.9601 & 0.9968 & 0.9974 \\
0.8 & 97.87 & 96.42 & 99.32 & 99.30 & 0.9578 & 0.9955 & 0.9965 \\
\hline 0.7 & 97.39 & 95.47 & 99.31 & 99.29 & 0.9486 & 0.9973 & 0.9978 \\
\hline 0.6 & 97.57 & 95.35 & 99.80 & 99.80 & 0.9524 & 0.9950 & 0.9964 \\
\hline
\end{tabular}

\section{Identification ability for new diseases}

Identifying potential diseases-associated with known miRNAs contributes to the study of pathological mechanisms of diseases. Similarly, we remove the disease from our dataset if its similarity value is greater than the threshold: $0.7,0.6,0.5,0.4,0.3$ and 0.2 , respectively. Please note that the highest similarity score is 0.7370 in our dataset, and only 290 positive samples remained when the threshold is set to 0.1. Finally, we constructed 6 non-redundant datasets, and the corresponding results were shown in Table 2. As the threshold decreases from 0.7 to 0.2 , all indicators change slightly around a certain value. For example, Acc and Sen fluctuate slowly around the average of $97.81 \%$ and $96.34 \%$. Even if the threshold is reduced to 0.2, our method still achieves Spe of 98.68\%, Pre of 98.61\%, Mcc of 0.9469, AUROC of 0.9934 and AUPRC of 0.9942. These results demonstrate that our method can identify potential miRNA-related diseases.

Table 2 The results of different non-redundant datasets 


\begin{tabular}{llllllll}
\hline Threshold & Acc(\%) & Sen(\%) & Spe(\%) & Pre(\%) & Mcc & AUROC & AUPRC \\
\hline 0.7 & 97.89 & 96.40 & 99.37 & 99.35 & 0.9582 & 0.9958 & 0.9967 \\
0.6 & 98.03 & 96.51 & 99.55 & 99.54 & 0.9609 & 0.9955 & 0.9963 \\
\hline 0.5 & 97.97 & 96.52 & 99.43 & 99.41 & 0.9598 & 0.9974 & 0.9979 \\
\hline 0.4 & 97.90 & 96.41 & 99.40 & 99.38 & 0.9550 & 0.9962 & 0.9975 \\
\hline 0.3 & 97.73 & 96.13 & 99.31 & 99.30 & 0.9550 & 0.9977 & 0.9981 \\
\hline 0.2 & 97.33 & 96.04 & 98.68 & 98.61 & 0.9469 & 0.9934 & 0.9942 \\
\hline
\end{tabular}

\section{Prediction capability for potential miRNA-disease associations}

To further verify the robustness of our method, we construct a series of non-redundant datasets according to the different thresholds following these steps:: (1) Set a threshold, and randomly select a miRNA-disease association involved in the positive sample set. (2) Calculate the similarity between the selected association and other associations contained in the positive sample set. (3) Remove the selected miRNA-disease association if its similarity value is higher than the specific threshold, otherwise, keep it in the positive sample set. (4) Repeat step 3, until the similarity value of any two association pairs is lower than the threshold, and the obtained set is called as non-redundant positive sample set. (5) Randomly choose an association from reliable negative samples, and calculate its similarity values with each association in the positive sample set as well as in the negative sample set. (6) Delete the chosen association from the reliable negative samples if similarity values are higher than the threshold, otherwise, remain it. The obtained set is known as non-redundant negative sample set. (7) Repeat step 6, until the size of the non-redundant positive sample set and non-redundant negative sample set is equal. (8) Combine the non-redundant positive sample set with the non-redundant negative sample set to construct the non-redundant training dataset. Finally, we set two thresholds 0.9 and 0.8 to build the two non-redundant training datasets (Only 53 samples contained in non-redundant negative sample set when the threshold is set as 0.7 , it is unable to construct the non-redundant training dataset with positive-negative ratio 1:1.). Results of five-fold cross-validation test are shown in Table 3. 
The Acc of $97.88 \%$, Sen of $96.44 \%$, Spe of $99.33 \%$, Pre of $99.31 \%$, Mcc of 0.9581 , AUROC of 0.9964 and AUPRC of 0.9973 are obtained when the threshold is set to 0.9. The Acc, Sen, Spe, Pre, Mcc, AUROC and AUPRC only decrease 0.29\%, 0.52\%, 0.06\%, 0.07\%, 0.0057, 0.0023 and 0.0015 , when change the threshold from 0.9 to 0.8 . There results demonstrate that the current method has a prominent robustness and can identify potential miRNAdisease associations.

Table 3 The results of 5-fold cross-validation based on the two non-redundant miRNAdisease associations datasets.

\begin{tabular}{llllllll}
\hline Threshold & Acc(\%) & Sen(\%) & Spe(\%) & Pre(\%) & Mcc & AUROC & AUPRC \\
\hline 0.9 & 97.88 & 96.44 & 99.33 & 99.31 & 0.9581 & 0.9964 & 0.9973 \\
0.8 & 97.59 & 95.92 & 99.27 & 99.24 & 0.9524 & 0.9941 & 0.9958 \\
\hline
\end{tabular}

\section{Comparison with other methods}

We further compare our method with three existing models PBMDA [22], WBSMDA [18] and RLSMDA [24] by constructing a new dataset including 4119 known associations between 415 miRNAs and 327 diseases from HMDD (v2.0). For PBMDA, we downloaded the code from its supplementary material to obtain the prediction results. For WBSMDA and RLSMDA, we rewritten the code based on their articles. The three approaches have been confirmed to achieve excellent prediction accuracy. The ROC and PRC curves of various methods were shown in Fig. 6. After implemented five-fold cross-validation, SeqSymRF achieved the average AUROC and ARPRC were 0.9640 and 0.9704 . The AUROC and AUPRC of PBMDA, RLSMDA and WBSMDA were 0.9189 and 0.9211, 0.8328 and 0.7964, 0.8293 and 0.8346 , respectively. The AUROC of current method was $4.51 \%, 13.12 \%$ and 13.47\% higher than those of three models (PBMDA, RLSMDA and WBSMDA), and AUPRC was $4.93 \%, 17.40 \%$ and $13.94 \%$ improvement. In conclusion, Seq-SymRF is an effective and accurate method for predicting miRNA-disease associations. 
[Figure 5]

\section{Case Study}

To further evaluate the prediction performance and demonstrate application ability of our method, we implement the following two case studies: (1) two common diseases breast neoplasms and leukemia are removed from our dataset and predict their association score with each miRNA. (2) We choose a miRNA hsa-mir-21 un-included in the dataset and predict its association score with each disease. Top 50 results of case study are validated by dbDEMC [30] or the literature.

Breast cancer is one of the most fatal diseases among female. According to the Global Cancer Statistics 2018 [31], female breast cancer is the second most common cancer, accounting for $18.6 \%$ of cancer deaths, behind lung cancer. MiRNA has practical effect on the treatment and diagnosis of breast cancer. For example, miRNA-223 can maintain cell proliferation of breast cancer cell through targeting FOXO1 [32] and let-7a can inhibit growth and migration of breast cancer cell by targeting HMGA1 [33]. Taking breast neoplasms as case study, our random forest model was implemented to prioritize candidate miRNAs. Table 4 lists the prediction score with different miRNA. From the table, we can see that 33 of top 50 potential related miRNAs were confirmed to be associated with breast neoplasms based on the dbDEMC database.

\section{Table 4 here}

Leukemia is a group of life-threatening malignant disorders of the blood and bone marrow. It is a dangerous disease which may occur at all ages, from the newborn to the very old [34]. As a special biomarker, miRNA also has a significant impression in leukemia treatment. The identification of potential association between miRNA and leukemia is very helpful in the development of therapeutic drugs. The prediction scores between leukemia and each candidate miRNA are listed in Table 5. In our model, 36 of top 50 potential related miRNAs were confirmed based on the dbDEMC database. 
Mir-21 is a well know miRNA, and it has been proved to be associated with many diseases, especially cancer. Its association scores with various diseases are predicted and listed in Table 6 . We can see that 32 of top 50 potential associations were confirmed based on the literature.

\section{Table 6 here}

\section{Discussion}

As more miRNAs are discovered, more and more evidence indicates that miRNAs are closely related to the occurrence and development of various human diseases. Using computational method to identify the miRNA-disease associations can help not only reduce time and experiment consumption, but also accelerate drug research and development. In this research, we used cross validation to evaluate prediction performance by constructing a series of dataset. Case studies are also conducted by applying Seq-SymRF on several important human diseases and miRNAs. For breast neoplasms, leukemia and hsamir- $21,66 \%, 72 \%, 64 \%$ of the predicted miRNA-disease associations in the top 50 are confirmed by database and literature, respectively. The outstanding performance of our model dependent on three reasons: (1) we constructed the dataset based on the HMDD which collects the newest experiment confirmed miRNA-disease associations. (2) Instead of selecting the negative sample randomly, we calculate the Euclidean distance between the un-labeled sample and the positive cluster center, and select the reliable negative sample. (3) The miRNA sequence features and disease symptom descriptors are used to characterize miRNA and disease, respectively. (4) The random forest classifier is a powerful model and able to complete the binary classification task very easily and efficiently. Of course, our method also has some limitations. For example, after integrate HMDD and symptom-disease network, only a part of disease in HMDD are choose. Similarly, just a part of miRNAs are collected. The incompleteness of miRNAs and diseases affects the performance of the model. After solving the limitation and integrating more information, we believe that the performance of Seq-SymRF model can be further improved.

\section{Conclusion}

The miRNA sequence and disease symptom play a significant role. Treating them as the feature to train the random forest model is a relatively new approach. Compared with traditional methods, it contains more biological and medical information. We implement the Seq-SymRF model to infer the miRNAdisease associations based on the two features and achieve an excellent performance. From different experimental results, it can be seen that the Seq-SymRF could be a helpful and reliable model to explore the complex relationship between miRNAs and diseases.

\section{Methods}




\section{MiRNA characterization}

Different from existing methods, we used the sequence information of miRNA to replace the functional similarity and Gaussian interaction profile kernel similarity. Firstly, we retrieved 1917 human miRNA hairpin sequences from miBase database. Secondly, miRNA sequence secondary structure was predicted through the web-server RNAfold [35] and the secondary structure with dot-bracket notation and the minimum free energy was obtained. In the dot-bracket notation, "("and ")" represent the paired nucleotide local near in the 5 '-end and 3 '-end, respectively. The dot "." represents the unpaired one. In the work, we use "(" for both paired situations. Thirdly, the 10 global and 32 local features were calculated. The global features contain symmetric difference, number of base pairs, GC content, length base pair ratio (length of the sequence/the number of base pairs), sequence length, length of central loop, free energy per nucleotide, bulge number, tail length and the number of tail(s). The local feature means 8 possible

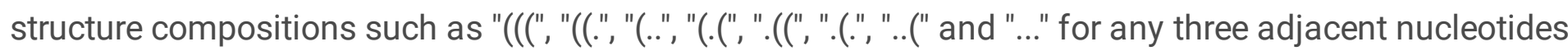
[36]. There are 4 kinds of bases $(A, U, C, G)$ in the middle position to constitute 32 different combination $(8 \times 4)$. In addition, the statistical values of 84 nucleotide ( 4 base count, 16 dimer count and 64 codon count) were also calculated. Finally, a feature vector with $126(10+32+84)$ dimensions containing global, local and nucleotide statistics count was employed to characterize miRNA sequence.

\section{Disease characterization}

Symptoms are abnormal states occurrence when the body has a lesion. One symptom can associate with different diseases, and vice versa. In the daily treatment of the hospital, through a comprehensive analysis of the symptoms, doctor can make a preliminary understanding of the patient's state and determine further treatment options. Thus, clinical symptoms were utilized to characterize disease instead of semantic similarity and Gaussian interaction profile kernel similarity. The associations between disease and their clinical symptoms were retrieved from the human symptom-disease network [37], in which association scores were calculated by using the term frequency-inverse document frequency algorithm and term frequency was replaced by the absolute co-occurrence. Finally, each of diseases can be characterized through a feature vector with 322 dimensions, in which every element corresponding to one specific symptom and was encoded as a value larger than or equal to zero for explaining the association strength between disease and symptom.

\section{Human miRNA-disease associations characterization}

In this study, we downloaded the human miRNA-disease associations data from the HMDD database (v3.0) constructed by Huang et al., [38]. A total of 18733 experimentally confirmed associations between 1208 miRNAs and 894 diseases were obtained. After removing these associations, in which miRNA and disease were not involved in the miBase database and the symptom-disease network, a benchmark dataset containing 7456 associations between 917 miRNAs and 339 diseases was obtained. Anew feature vector with $448(126+322)$ dimensions was obtained by concatenating miRNA feature vector and disease feature vector, and utilized to characterize each miRNA-disease association pair.

\section{Negative sample selection}

Page 14/ 26 
Identifyication of miRNA-disease association is a binary classification problem, i.e. infer whether a miRNA is associated with a disease. Unfortunately, no database is currently dedicated to collecting negative samples. Therefore, we treat our method as a positive-unlabeled learning and select the reliable negative sample from the unlabeled sample (i.e. un-included in the HMDD database). All experiment confirmed associations were considered as positive set (i.e. the constructed benchmark dataset), and remaining $303407(917 \times 339-7456)$ associations were regarded as the unlabeled set. We selected the reliable negative sample following the steps: (1) Sum up all the sample in positive set with each dimension and calculate their mean to form a standard 448 dimension vector as the cluster center. (2) Calculate the Euclidean distance between each sample in unlabeled set and cluster center. (3) Calculate the average Euclidean distance (AOD). (4) Treat the sample in un-labeled set as reliable negative sample if its Euclidean distance is higher than AOD. (5) Generate a reliable negative sample dataset.

\section{Model construction}

Random forest, a classifier containing multiple decision trees, has achieved admirable performance in different fields. In this study, we constructed a random forest classifier model to predict the potential miRNA-disease associations.

Figure 6. Flowchart of the current method.

\section{Abbreviations}

Acc:accuracy; Spe:specificity; Sen:sensitivity; Pre:precision; Mcc:Matthews correlation coefficient; AUROC:Area under the receiver-operating characteristic curve; AUPRC:Area under the precision-recall curve; mDPS:miRNA-disease pair similarity; HMDD:Human microRNA disease database; dbDEMC:Database of differentially expressed miRNAs in human cancers; miBase:the microRNA database; MeSH:Medical subject heading; AOD:average Euclidean distance; RSD:relative standard deviation; NCBI:National Center for Biotechnology Information

\section{Declarations}

\section{Ethics approval and consent to participate}

Not applicable.

\section{Consent for publication}

Not applicable.

\section{Availability and Requirements}

Project name: Seq-SymRF

Project home page: https://github.com/LeeKamlong/Seq-SymRF 
Operating system(s): Windows

Programming language: Matlab

Other requirements: Matlab2018b or higher

License: GNU GPL.

Any restrictions to use by non-academics: None

\section{Availability of data and materials}

Seq-SymRF is available at https://github.com/LeeKamlong/Seq-SymRF

\section{Competing interests}

The authors declare that they have no competing interests.

\section{Funding}

This work, including publication costs, was supported by National Natural Science Foundation of China (No.21675035; No.21675180; No.21775169). This work was also supported by the Scientific Technology Project of Guangdong Province (2015A030401033; 2016B010108007; 2016A010103039; 2017B020221001); Special Innovation Project of University in Guangdong Province (2018KTSCX109); Special Funds of Key Disciplines Construction from Guangdong and Zhongshan Cooperating, and the Scientific Technology Project of Guangzhou City (201604020145). These funding bodies did not play any role in the design of study, the interpretation of data, or the writing of this manuscript.

\section{Authors' contributions}

JLL collected the data, experimented and drafted the manuscript. JLL provides experimental ideas and revised manuscripts. XYC, QXH, YW, YX and ZD participated in the discussion of this work. ZCL and XYZ also participated in the discussion and revision of the manuscript. All authors read, commented and approved the final manuscript.

\section{Acknowledgements}

Not applicable.

\section{Author details}

${ }^{1}$ School of Chemistry and Chemical Engineering, Guangdong Pharmaceutical University, Guangzhou, 510006, People's Republic of China

${ }^{2}$ School of Chemistry, Sun Yat-Sen University, Guangzhou, 510275, People's Republic of China 
${ }^{3}$ Key Laboratory of Digital Quality Evaluation of Chinese Materia Medica of State Administration of Traditional Chinese Medicine, Guangzhou, 510006, People's Republic of China

\section{References}

1. Ambros V. The functions of animal microRNAs. Nature. 2004;431:350-355.

2. Vasudevan S, Tong YC, Steitz JA. Switching from Repression to Activation: MicroRNAs Can UpRegulate Translation. Science. 2007;318:1931-1934.

3. Cheng AM, Byrom MW, Shelton J, Ford LP. Antisense inhibition of human miRNAs and indications for an involvement of miRNA in cell growth and apoptosis. Nucleic Acids Res. 2005;33:1290-1297.

4. Karp X, Ambros V. Encountering microRNAs in cell fate signaling. Science. 2005;310:1288-1289.

5. Miska EA. How microRNAs control cell division, differentiation and death. Curr. Opin. Genet. Dev. 2005;15:563-568.

6. Xu PZ, Guo M, Hay BA. MicroRNAs and the regulation of cell death. Trends Genet. 2004;20:617-624.

7. Alshalalfa M, Alhajj R. Using context-specific effect of miRNAs to identify functional associations between miRNAs and gene signatures. BMC bioinformatics. 2013;14 Suppl 12:S1.

8. Bartel DP. MicroRNAs: target recognition and regulatory functions. Cell. 2009;136:215-233.

9. Cui QH, Yu ZB, Purisima EO, Wang E. Principles of microRNA regulation of a human cellular signaling network. Mol. Syst. Biol. 2006;2:46.

10. Su H, Li X, Luo X, Chen Y. Functions of miRNA in animal viral infection. J. Anim. Vet. Adv. 2013;12(14):1202-1209.

11. Huang Y, Yang YB, Zhang XH, Yu XL, Wang ZB, Cheng XC. MicroRNA-21 gene and cancer. Med Oncol. 2013;30:376.

12. Yang YD, An R, Feng T, Qin XF, Zhang J, Bo YL, Niu B. Skullcapflavone I suppresses proliferation of human lung cancer cells via down-regulating microRNA-21. Exp. Mol. Pathol. 2019;110:104285.

13. Lee RC, Feinbaum RL, Ambros V. The C. elegans heterochronic gene lin- 4 encodes small RNAs with antisense complementarity to lin-14. Cell. 1993;75(5): 843.

14. Reinhart BJ, Slack FJ, Basson M, Pasquinelli AE, Bettinger JC, Rougvie AE, Horvitz HR, Ruvkun G. The 21-nucleotide let-7 RNA regulates developmental timing in Caenorhabditis elegans. Nature. 2000;403:901-906.

15. Kozomara, Birgaoanu M, Griffiths-Jones S. miRBase: from microRNA sequences to function. Nucleic Acids Res. 2019;47:155-162.

16. Chen X, Xie D, Zhao Q, You ZH. MicroRNAs and complex diseases: from experimental results to computational models. Brief. Bioinform. 2019;20(2):515-539.

17. Jiang Q, Hao Y, Wang G, Juan L, Zhang T, Teng M, Liu Y, Wang Y. Prioritization of disease microRNAs through a human phenome-microRNAome network. BMC Syst. Biol. 2010;4 Suppl 1: S2. 
18. Chen X, Yan CC, Xu Z, You ZH, Deng L, Liu Y, Zhang Y, Dai Q. WBSMDA: Within and Between Score for MiRNA-Disease Association prediction. Sci. Rep. 2016;6:21106.

19. Chen X, Liu MX, Yan GY. RWRMDA: predicting novel human microRNA-disease associations. Mol. BioSyst. 2012;8:2792-2798.

20. Xuan P, Han K, Guo Y, Li J, Li X, Zhong Y, Zhang Z, Ding J. Prediction of potential disease-associated microRNAs based on random walk. Bioinformatics. 2015;31(11):1805-1815.

21. Chen X, Yan CC, Zhang X, You ZH, Huang YA, Yan GY. HGIMDA: Heterogeneous graph inference for miRNA-disease association prediction. Oncotarget. 2016;7(40):65257-65269.

22. You ZH, Huang ZA, Zhu Z, Yan GY, Li ZW, Wen Z, Chen X. PBMDA: A novel and effective path-based computational model for miRNA-disease association prediction. PLoS Comput Biol. 2017;13 (3):e1005455.

23. Xu J, Li CX, Lv JY, Li YS, Xiao Y, Shao TT, et al. Prioritizing candidate disease miRNAs by topological features in the miRNA target-dysregulated network: case study of prostate cancer. Mol. Cancer Ther. 2011;10:1857-1866.

24. Chen X, Yan GY. Semi-supervised learning for potential human microRNA-disease associations inference. Sci. Rep. 2014;4:5501.

25. Mørk S, Pletscher-Frankild S, Caro AP, Gorodkin J, Jensen LJ. Protein-driven inference of miRNAdisease associations. Bioinformatics. 2014;30:392-397.

26. Shi HB, Xu J, Zhang GD, Xu LD, Li CQ, Wang L, Zhao Z, Jiang W, et al. Walking the interactome to identify human miRNA-disease associations through the functional link between miRNA targets and disease genes. BMC Syst. Biol. 2013;7:101.

27. Wang D, Wang J, Lu M, Song F, Cui Q. Inferring the human microRNA functional similarity and functional network based on microRNA-associated diseases, Bioinformatics. 2010;26(13):16441650.

28. KNOTTNERUS JA. The effects of disease verification and referral on the relationship between symptoms and diseases. Med. Decis. Making. 1987;7;139-148.

29. Huang Y, Niu B, Gao Y, Fu L, Li W. CD-HIT Suite: a web server for clustering and comparing biological sequences. Bioinformatics. 2010;26 (5):680-682.

30. Yang Z, Wu L, Wang A, Tang W, Zhao Y, Zhao H, Teschendorff AE. dbDEMC 2.0: updated database of differentially expressed miRNAs in human cancers. Nucleic Acids Res. 2017;45(D1):D812-D818.

31. Bray F, Ferlay J, Soerjomataram I, Siegel RL, Torre LA, Jemal A. Global Cancer Statistics 2018: GLOBOCAN Estimates of Incidence and Mortality Worldwide for 36 Cancers in 185 Countries. CA: Cancer J Clin. 2018;68:394-424.

32. Wei YT, Guo DW, Hou XZ, Jiang DQ. MiRNA-223 suppresses FOX01 and functions as a potential tumor marker in breast cancer. Cell. Mol. Biol. (Noisy-le-Grand, France). 2017;63(5):113.

33. Liu K, Zhang C, Li T, Ding Y, Tu T, Zhou F, Qi W, Chen H, Su X. Let-7a inhibits growth and migration of breast cancer cells by targeting HMGA1. Int. J. Oncol. 2015;46:2526-2534. 
34. An Q, Fan $\mathrm{CH}$, Xu SM. Recent perspectives of pediatric leukemia-an update. Eur. Rev. Med. Pharmacol. Sci. 2017;21 Suppl 4:31-36.

35. Lorenz R, Bernhartand SH, Höner zu Siederdissen C, Tafer H, Flamm C, Stadler PF, Hofacker IL. ViennaRNA Package 2.0. Algorithms Mol. Biol. 2011;6:26.

36. Xue CH, Li F, He T, Liu GP, Li YD, Zhang XG. Classification of real and pseudo microRNA precursors using local structure-sequence features and support vector machine. BMC bioinformatics. 2005;6:310.

37. Zhou X, Menche J, Barabási AL, Sharma A. Human symptoms-disease network. Commun. 2014;5:4212.

38. Huang Z, Shi J, Gao Y, Cui C, Zhang S, Li J, Zhou Y, Cui Q. HMDD v3.0: a database for experimentally supported human microRNA-disease associations. Nucleic Acids Res. 2019;47(D1):D1013-D1017.

\section{Tables}

Table 4 Prediction list of the top 50 prioritized miRNAs associated with breast neoplasms 


\begin{tabular}{|c|c|c|c|c|c|}
\hline & core & Evidence & $\mathrm{miF}$ & core & $\mathrm{Ev}$ \\
\hline hsa. & 98 & C & hsa & 96 & un \\
\hline hsa- & 98 & MC & 410 & 0.96 & \\
\hline hsa-r & 0.98 & & $\mathrm{~b}$ & 0.96 & \\
\hline hsa-r & 0.98 & $d$ & hsa & 0.96 & \\
\hline hsa-n & 0.97 & & hsa & 0.96 & \\
\hline hsa-1 & 0.97 & & hsa & 0.96 & \\
\hline hsa-1 & 0.97 & & hsa & 0.96 & \\
\hline hsa-1 & 0.96 & & hsa & 0.96 & unc \\
\hline hsa- & 0.96 & dbDEMC & -3170 & 0.96 & $\mathrm{dl}$ \\
\hline hsa-I & 0.96 & dbDEMC & hsa- & 0.96 & uncor \\
\hline hsa-mir-31 & 0.96 & dbDEMC & hsa- & 0.95 & $\mathrm{db}$ \\
\hline hsa- & 0 a & MC & hsa-l & 0.95 & unconf \\
\hline hsa-r & 96 & MC & hsa & 0.5 & $\mathrm{dl}$ \\
\hline hsa & 96 & dbDEMC & hsa- & 0.95 & un \\
\hline hsa- & 96 & MC & hsa & 0. & $\mathrm{db}$ \\
\hline hsa-mir- & 0.96 & unconfirmed & hsa-mir-27a & 0.95 & dbDEMC \\
\hline hsa-mir-153-2 & 0.96 & unconfirmed & hsa-mir-101-1 & 0.95 & unconfirmed \\
\hline hsa-mir-125a & 0.96 & dbDEMC & hsa-mir-148a & 0.95 & UNDEIVC \\
\hline hsa-mir-125b-2 & 0.96 & unconfirmed & hsa-mir-224 & 0.95 & dbDEMC \\
\hline hsa-mir-129-2 & 0.96 & unconfirmed & hsa-mir-23b & 0.95 & dbDEMC \\
\hline hsa-mir-195 & 0.90 & dbDEMC & hsa-mir-142 & 0.95 & unconfirmed \\
\hline hsa-n & 96 & dbDEMC & hsa & 0.95 & $\mathrm{dbD}$ \\
\hline hsa-mir-155 & 96 & & hsa-mir-126 & 0.95 & \\
\hline hsa-mir-34c & 0.96 & & hsa-mir-146a & 0.95 & \\
\hline hsa-mir-302d & 0.96 & dbDEMC & hsa-mir-184 & 0.95 & dbDEMC \\
\hline
\end{tabular}

Table 5 Prediction list of the top 50 prioritized miRNAs associated with leukemia 


\begin{tabular}{|c|c|c|c|c|c|}
\hline miRNA & Score & Evidence & miRNA & Score & Evidence \\
\hline hsa-mir-21 & 0.92 & dbDEMC & hsa-mir-489 & 0.88 & unconfirmed \\
\hline hsa-mir-146a & 0.92 & dbDEMC & hsa-mir-505 & 0.88 & dbDEMC \\
\hline hsa-mir-142 & 0.91 & dbDEMC & hsa-mir-449c & 0.88 & unconfirmed \\
\hline hsa-let-7a-1 & 0.91 & unconfirmed & hsa-mir-26b & 0.88 & dbDEMC \\
\hline hsa-mir-19a & 0.90 & dbDEMC & hsa-mir-106a & 0.88 & dbDEMC \\
\hline hsa-mir-20a & 0.90 & dbDEMC & hsa-mir-16-1 & 0.87 & unconfirmed \\
\hline hsa-mir-494 & 0.90 & dbDEMC & hsa-mir-19b-1 & 0.87 & dbDEMC \\
\hline hsa-let-7b & 0.89 & dbDEMC & hsa-mir-31 & 0.87 & dbDEMC \\
\hline hsa-mir-17 & 0.89 & dbDEMC & hsa-mir-101-1 & 0.87 & unconfirmed \\
\hline hsa-mir-22 & 0.89 & dbDEMC & hsa-mir-511 & 0.87 & unconfirmed \\
\hline hsa-mir-26a-1 & 0.89 & unconfirmed & hsa-mir-23b & 0.87 & dbDEMC \\
\hline hsa-mir-145 & 0.89 & dbDEMC & hsa-let-7c & 0.86 & dbDEMC \\
\hline hsa-mir-125b-2 & 0.89 & unconfirmed & hsa-mir-199a-1 & 0.86 & dbDEMC \\
\hline hsa-mir-30c-1 & 0.89 & dbDEMC & hsa-mir-125b-1 & 0.86 & dbDEMC \\
\hline hsa-mir-26a-2 & 0.89 & dbDEMC & hsa-mir-449a & 0.86 & unconfirmed \\
\hline hsa-mir-93 & 0.89 & dbDEMC & hsa-let-7a-3 & 0.85 & unconfirmed \\
\hline hsa-mir-194-1 & 0.89 & unconfirmed & hsa-mir-15a & 0.85 & dbDEMC \\
\hline hsa-let-7f-1 & 0.88 & $\mathrm{dbDEMC}$ & hsa-mir-32 & 0.85 & dbDEMC \\
\hline hsa-mir-92a-2 & 0.88 & unconfirmed & hsa-mir-153-2 & 0.85 & unconfirmed \\
\hline hsa-mir-224 & 0.88 & dbDEMC & hsa-mir-125a & 0.85 & dbDEMC \\
\hline hsa-mir-122 & 0.88 & unconfirmed & hsa-mir-195 & 0.85 & dbDEMC \\
\hline hsa-mir-126 & 0.88 & dbDEMC & hsa-mir-206 & 0.85 & dbDEMC \\
\hline hsa-mir-150 & 0.88 & dbDEMC & hsa-mir-106b & 0.85 & dbDEMC \\
\hline hsa-mir-29c & 0.88 & dbDEMC & hsa-mir-34b & 0.85 & dbDEMC \\
\hline hsa-mir-130b & 0.88 & dbDEMC & hsa-mir-34c & 0.85 & dbDEMC \\
\hline
\end{tabular}

Table 6 Prediction list of the top 50 prioritized disease associated with hsa-mir-21 


\begin{tabular}{|c|c|c|c|c|c|}
\hline disease & Score & Evidence & disease & Score & Evidence \\
\hline Coronary Artery & 0.99 & PMID: & Epilepsy & 0.95 & PMID: \\
\hline Disease & & 25656948 & & & 24560344 \\
\hline \multirow[t]{2}{*}{ Glioma } & 0.98 & PMID: & Colitis, Ulcerative & 0.95 & PMID: \\
\hline & & 26701969 & & & 30530166 \\
\hline Nephrotic & 0.98 & PMID: & Lichen Planus & 0.95 & PMID: \\
\hline Syndrome & & 24992527 & & & 29480379 \\
\hline Odontogenic & 0.98 & unconfirmed & Cleidocranial & 0.95 & unconfirmed \\
\hline Tumors & & & Dysplasia & & \\
\hline \multirow[t]{2}{*}{ Cataract } & 0.98 & PMID: & Silicosis & 0.95 & PMID: \\
\hline & & 27586871 & & & 26784015 \\
\hline \multirow[t]{2}{*}{ Hyperglycemia } & 0.98 & PMID: & Leiomyoma & 0.95 & PMID: \\
\hline & & 28627440 & & & 29747655 \\
\hline Lymphoma, Non- & 0.98 & PMID: & Lyme Disease & 0.95 & unconfirmed \\
\hline Hodgkin & & 29067124 & & & \\
\hline \multirow[t]{2}{*}{ Retinoblastoma } & 0.97 & PMID: & Guillain-Barre & 0.95 & unconfirmed \\
\hline & & 27600360 & Syndrome & & \\
\hline \multirow[t]{3}{*}{ Lymphoma } & 0.97 & unconfirmed & Muscular & 0.94 & PMID: \\
\hline & & & Dystrophy, & & 30692507 \\
\hline & & & Duchenne & & \\
\hline \multirow[t]{2}{*}{ Lymphoma, T-Cell } & 0.97 & PMID: & Mycosis & 0.94 & PMID: \\
\hline & & 27329723 & Fungoides & & 25503151 \\
\hline Hyperuricemia & 0.97 & unconfirmed & Alopecia & 0.94 & unconfirmed \\
\hline \multirow[t]{2}{*}{ Osteonecrosis } & 0.97 & PMID: & Diabetes Mellitus, & 0.94 & PMID: \\
\hline & & 30588193 & Type 1 & & 24937532 \\
\hline Pelvic Organ & 0.97 & unconfirmed & Carcinoma, & 0.94 & PMID: \\
\hline Prolapse & & & Embryonal & & 24977712 \\
\hline Carcinoma, Renal & 0.96 & PMID: & Nevus & 0.94 & unconfirmed \\
\hline Cell & & 27814278 & & & \\
\hline
\end{tabular}


Leukemia,

0.96

PMID

Aortic Aneurysm

0.94

PMID:

Promyelocytic, 20143188

29519942

Acute

Hirschsprung

0.96

unconfirmed

Leukemia,

0.94

PMID:

Disease

Myeloid, Chronic-

25575817

Phase

Chondrosarcoma

0.96

unconfirmed

Prader-Willi

0.94 unconfirmed

Syndrome

\begin{tabular}{|c|c|c|c|c|c|}
\hline Huntington Disease & 0.96 & unconfirmed & $\begin{array}{l}\text { Digeorge } \\
\text { Syndrome }\end{array}$ & 0.94 & $\begin{array}{c}\text { PMID: } \\
26867589\end{array}$ \\
\hline Biliary Atresia & 0.96 & $\begin{array}{c}\text { PMID: } \\
26927196\end{array}$ & Anemia & 0.94 & $\begin{array}{c}\text { PMID: } \\
30657053\end{array}$ \\
\hline $\begin{array}{c}\text { Fatty Liver, } \\
\text { Alcoholic }\end{array}$ & 0.96 & unconfirmed & Osteosarcoma & 0.93 & $\begin{array}{c}\text { PMID: } \\
28742209\end{array}$ \\
\hline $\begin{array}{c}\text { Diabetic } \\
\text { Cardiomyopathies }\end{array}$ & 0.96 & $\begin{array}{c}\text { PMID: } \\
30180843\end{array}$ & Multiple Sclerosis & 0.93 & $\begin{array}{c}\text { PMID: } \\
30698680\end{array}$ \\
\hline Hearing Loss & 0.96 & $\begin{array}{c}\text { PMID: } \\
28224282\end{array}$ & Medulloblastoma & 0.93 & $\begin{array}{c}\text { PMID: } \\
29280516\end{array}$ \\
\hline Hypogonadism & 0.96 & unconfirmed & Choriocarcinoma & 0.93 & $\begin{array}{c}\text { PMID: } \\
27922982\end{array}$ \\
\hline Ganglioglioma & 0.95991453 & unconfirmed & Down Syndrome & 0.93 & unconfirmed \\
\hline Pheochromocytoma & 0.9575 & unconfirmed & $\begin{array}{l}\text { Coronavirus } \\
\text { Infections }\end{array}$ & 0.93 & unconfirmed \\
\hline
\end{tabular}

\section{Figures}


(A)

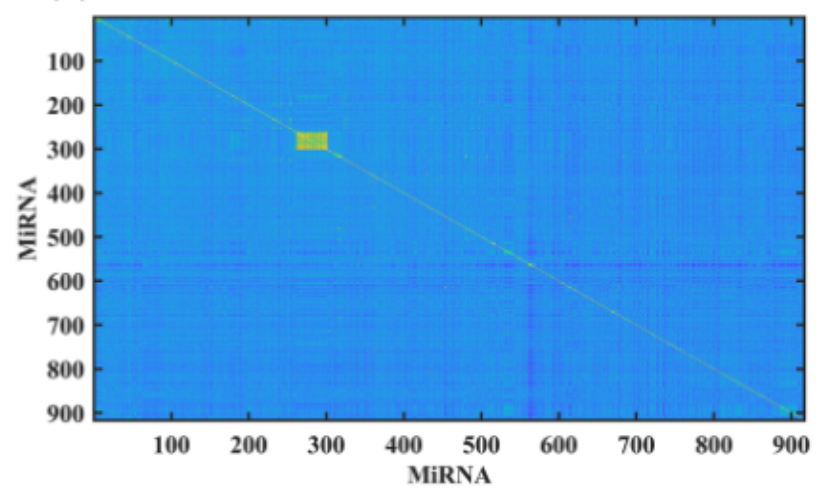

(C)

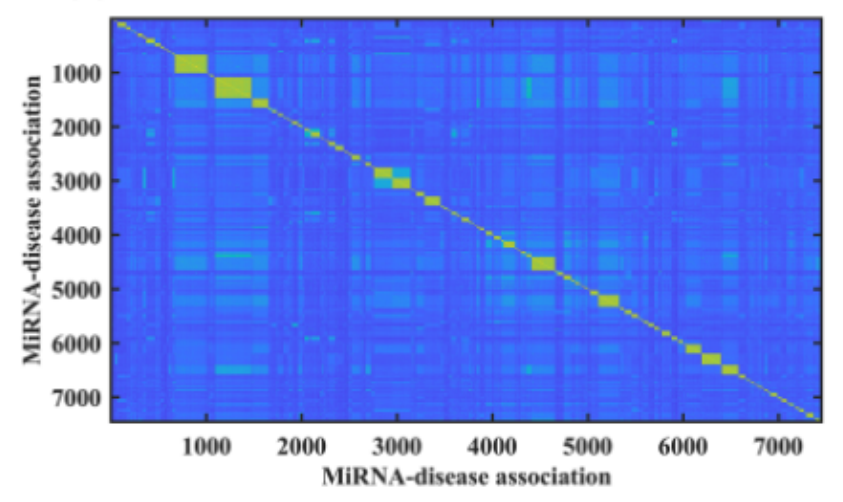

(B)

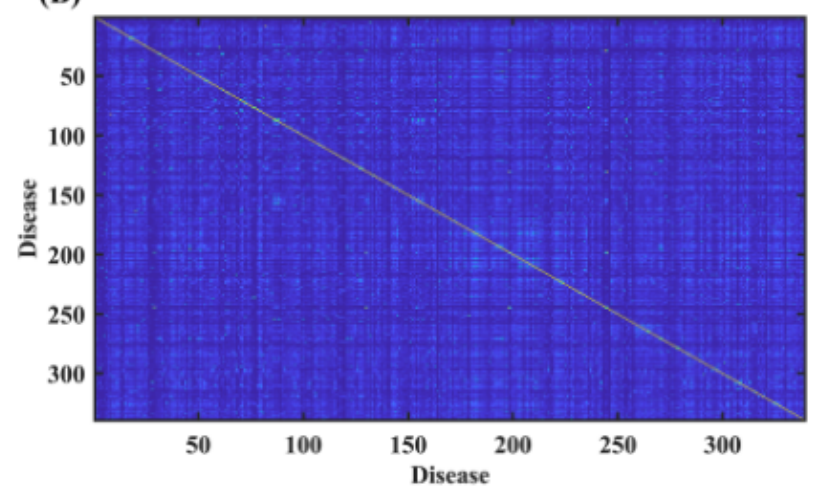

(D)

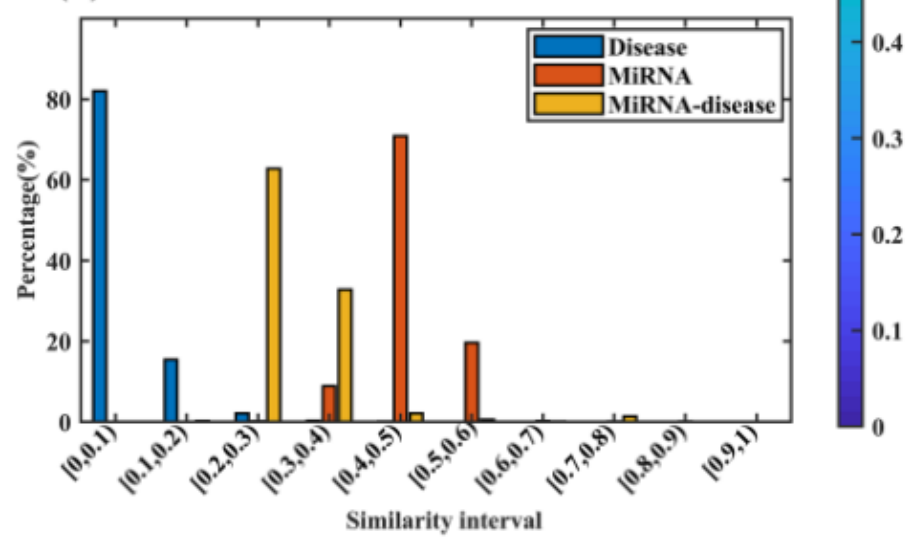

\section{Figure 1}

The similarity and statistical results of the benchmark dataset. (A), (B) and (C) describe the similarity for miRNA, disease and miRNA-disease association, respectively. (D) describes the distribution of miRNA, disease and miRNA-disease association similarity values at different intervals.

\section{Figure 2}

Comparison of different negative example selection strategy. $(A)$ describes the comparison of prediction results between two selection negative methods, and (B) describes the ROC and ROC for different selection strategy. In this figure, selecting $\mathrm{N}$ from $\mathrm{RN}$ was represented in blue and selecting $\mathrm{N}$ from UN was represented in red.

\section{Figure 3}

The comparison of 5 measures and two AUC among different threshold.

Figure 4 
The comparison results among different ratios of positive and negative samples.

\section{Figure 5}

Performance comparisons between Seq-SymRF and RLSMDA, WBSMDA as well as PBMDA.

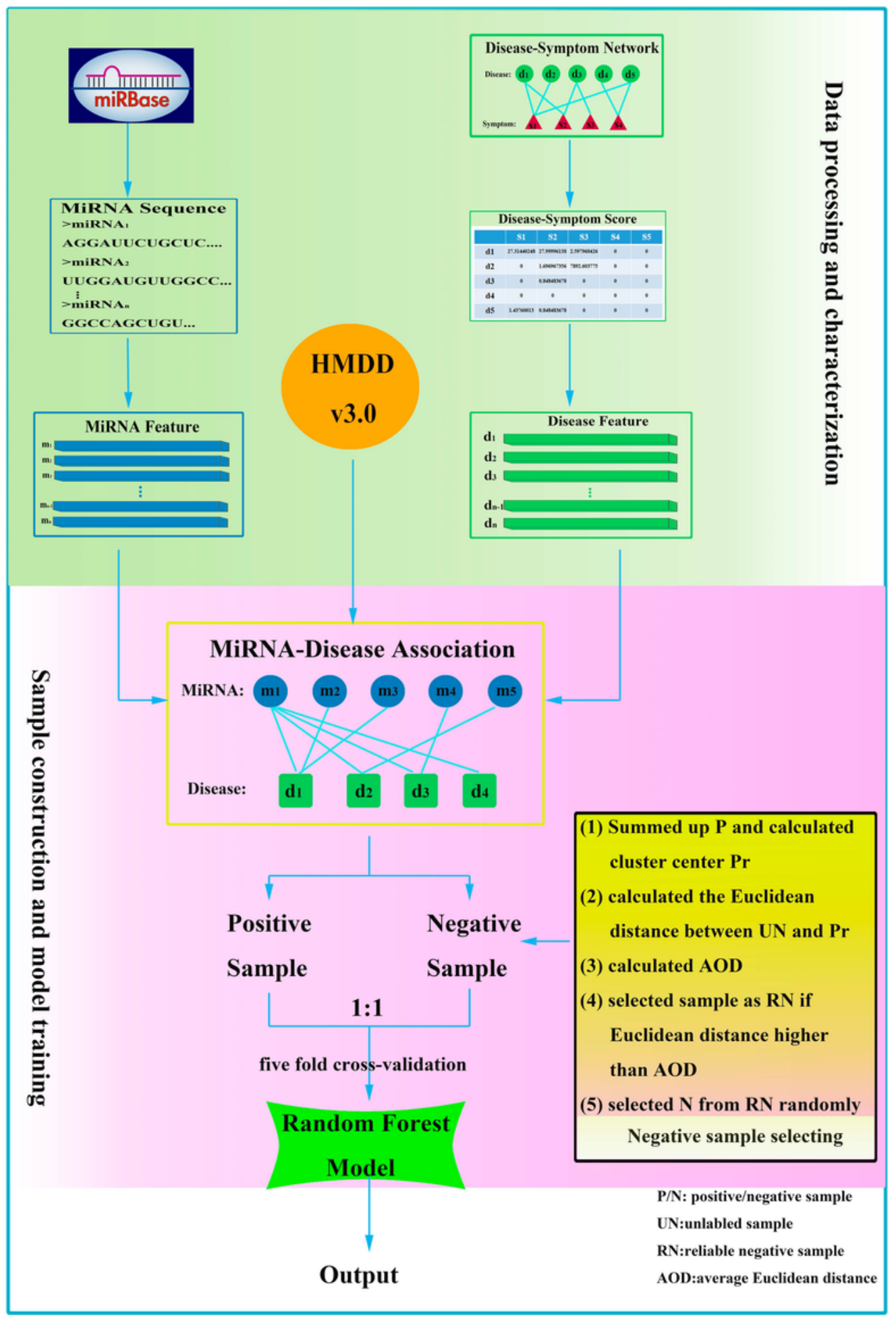

Figure 6 
Flowchart of the current method.

\section{Supplementary Files}

This is a list of supplementary files associated with this preprint. Click to download.

- Resultsformula.docx

- SupplementTable17.rar 